\title{
H2B ubiquitylation in transcriptional control: a FACT-finding mission
}

\author{
R. Nicholas Laribee, Stephen M. Fuchs, and Brian D. Strahl ${ }^{1}$ \\ Department of Biochemistry and Biophysics, University of North Carolina School of Medicine, Chapel Hill, \\ North Carolina 27599, USA
}

\begin{abstract}
A little more than 10 years ago, the laboratory of David Allis published two pioneering papers in Cell (Brownell et al. 1996; Mizzen et al. 1996) demonstrating that the transcriptional coactivators Gcn5 and $\mathrm{TAF}_{\mathrm{II}} 250$ (TAF1) could acetylate histones as part of their transcriptional activating functions. These studies fundamentally altered our understanding of transcriptional regulation and heralded a new era of research that has focused on understanding how covalent histone modifications regulate gene transcription. A recent flurry of studies has linked one modification in particular, histone H2B monoubiquitylation (H2Bub1), to the regulation of the elongation phase of the gene transcription cycle. A study in this issue of Genes \& Development from the Allis laboratory (Tanny et al. 2007) further strengthens this connection by showing that H2Bub1 functions in vivo to promote efficient RNA polymerase II (Pol II) elongation through chromatin. We will discuss the work up to this point that establishes H2Bub1 as a modification linked to transcriptional regulation, highlighting the importance of this current study, along with others, in shaping our understanding of how $\mathrm{H} 2 \mathrm{Bub} 1$ promotes transcription elongation through a chromatin landscape.
\end{abstract}

\section{A little goes a long way-H2B ubiquitylation as a 'trans'ient modification}

Protein ubiquitylation occurs through an enzymatic cascade involving three different factors plus ubiquitin. Initially, a ubiquitin activating enzyme (E1) creates an E1ubiquitin intermediate through the formation of a thioester bond in an ATP-dependent reaction (Pickart 2001). This activated form of ubiquitin is then transferred to a ubiquitin-conjugating (E2) enzyme that, in partnership with an E3 ubiquitin ligase, will mediate ubiquitin transfer to selective lysine residues on substrate proteins. Typically, polyubiquitylation signals a protein to be degraded via the $26 \mathrm{~S}$ proteasome, whereas monoubiquitylation modifies protein function (Pickart

${ }^{1}$ Corresponding author.

E-MAIL brian_strahl@med.unc.edu; FAX (919) 966-2852.

Article is online at http://www.genesdev.org/cgi/doi/10.1101/gad.1541507.
2001). H2Bub1 occurs at the Lys 123 position in budding yeast (Saccharomyces cerevisiae) and at the Lys 120 position (K120) in humans (Zhang 2003; Osley 2004). Although histones were the first proteins found to be ubiquitylated, research into the regulation and biological consequences of this modification on chromatin function languished, due in part to the difficulty in studying it. H2Bub1 exists at relatively low levels in vivo (accounting for $<10 \%$ of the total pool of $\mathrm{H} 2 \mathrm{~B}$ ) and is readily removed by the actions of ubiquitin proteases (Ubps) (Zhang 2003; Osley 2004). Using budding yeast as a model system, a group led by Mary Ann Osley showed that the E2 enzyme responsible for $\mathrm{H} 2 \mathrm{~B}$ monoubiquitylation in vivo was Rad6 (Robzyk et al. 2000). They further demonstrated that a $\mathrm{H} 2 \mathrm{~B}$ point mutant (K123R) that blocks monoubiquitylation shared the same mitotic and meiotic defects seen in a rad6s mutant, although it did not have the sensitivity to UV radiation that the rad $6 \Delta$ mutant exhibited (Robzyk et al. 2000). These results established Rad6 as a chromatin regulator and suggested that Rad6-mediated ubiquitylation of H2B plays an important role in both mitosis and meiosis.

Although H2Bub1 had been linked to such activities as increased transcription, DNA replication, mitosis, and meiosis, how this histone modification functioned in these processes remained unknown. New insights into the in vivo function of H2Bub1 occurred in 2002 when the Allis, Shilatifard, Strahl, and Struhl laboratories (Briggs et al. 2002; Dover et al. 2002; Ng et al. 2002; Sun and Allis 2002) determined that ubiquitylation of H2B by Rad6 regulated subsequent methylation of histone $\mathrm{H} 3$ at Lys 4 (H3K4me) and Lys 79 (H3K79me) through a "transtail" mechanism. Because H3K4me was found to demarcate the $5^{\prime}$ ends and coding regions of Pol II-transcribed genes, these results strongly suggested a role for H2Bub1 in transcriptional regulation (Bernstein et al. 2002; Santos-Rosa et al. 2002). This idea was supported by the fact that $\mathrm{H} 2 \mathrm{Bub} 1$ also regulates gene silencing in the heterochromatic-like regions in yeast (e.g., telomeres and rDNA) through its "trans-tail" regulation of $\mathrm{H} 3 \mathrm{~K} 4 \mathrm{me}$ and H3K79me, two histone modifications critical for this process (Briggs et al. 2001; Bryk et al. 2002; Dover et al. 2002; Sun and Allis 2002; van Leeuwen et al. 2002). Subsequent studies identified the Ring finger protein 
Bre1 as the E3 ligase that interacts with Rad6 to mediate H2Bub1 (Hwang et al. 2003; Wood et al. 2003a). Cells carrying a bre $1 \Delta$ mutation share many of the same phenotypes seen in a rad $6 \Delta$ mutant, including the transcriptional silencing defects caused by abolishment of H2Bub1 and loss of H3K4me and H3K79me. Lge1, another factor found to copurify with Bre1, also regulates $\mathrm{H} 2 \mathrm{Bub} 1$, but how it participates in $\mathrm{H} 2 \mathrm{~B}$ ubiquitylation is less understood (Hwang et al. 2003). H2Bubl was hypothesized to regulate the outcome of histone methylation by acting as either a "wedge" to open the local chromatin environment, thereby facilitating access of the methyltransferases to their substrates, or to serve as a "bridge" to recruit factors that promote the methylation event (Henry and Berger 2002; Sun and Allis 2002).

Initial efforts linking Rad6/Bre1 and H2Bub1 directly to the transcription process revealed that $\operatorname{Rad} 6$ is recruited to promoters in an activator- and Bre1-dependent manner. Additionally, Rad6 recruitment, and the establishment of H2Bub1, was found to be required for proper activation of inducible genes (Wood et al. 2003a; Kao et al. 2004). While these results provided an explanation for how both $\mathrm{H} 2 \mathrm{Bub} 1$ and $\mathrm{H} 3 \mathrm{~K} 4 \mathrm{me} 3$ could be established in the promoter and $5^{\prime}$ ends of genes, it left a fundamental question unanswered. How does H2Bub1, which also regulates $\mathrm{H} 3 \mathrm{~K} 4$ dimethylation $(\mathrm{H} 3 \mathrm{~K} 4 \mathrm{me} 2)$ and $\mathrm{H} 3 \mathrm{~K} 79$ di- and trimethylation (H3K79me2 and H3K79me3), get established within the gene body to regulate $\mathrm{H} 3 \mathrm{~K} 4 \mathrm{me}$ and H3K79me? This conundrum was resolved after the discovery that the PAF transcription elongation complex was essential for the regulation of $\mathrm{H} 2 \mathrm{Bub} 1(\mathrm{Ng}$ et al. 2003; Wood et al. 2003b; Xiao et al. 2005). This complex consists of five different proteins in yeast (Paf1, Rtf1, Ctr9, Cdc73, and Leo1) and was identified as a positive elongation factor that could associate with Pol II throughout the body of genes (Krogan et al. 2002; Mueller and Jaehning 2002; Kim et al. 2004). Individual deletions of these different members reduced H2Bub1 to varying degrees-from near abolishment in the $\operatorname{rtf} 1 \Delta$ and paf1 $\Delta$ strains to reduced levels in the $c t r 9 \Delta$ and $c d c 73 \Delta$ strains (Ng et al. 2003; Wood et al. 2003b; Xiao et al. 2005). Subsequent chromatin immunoprecipitation (ChIP) analyses revealed that Rad6, along with H2Bub1, could be detected within the ORF of multiple genes (Xiao et al. 2005). Significantly, the recruitment of Rad6, and the establishment of H2Bub1, to ORFs depended on the PAF complex. PAF also appears important for stimulating the activity of the Rad6 complex since in PAF deletion mutants, Rad6 could still be detected at the promoter regions of genes but no $\mathrm{H} 2 \mathrm{Bubl}$ was detected (Xiao et al. 2005). The interactions between Rad6 and PAF suggested an elegant mechanism in which Rad6 at promoter regions could be "handed off" to the elongating Pol II complex via interactions with PAF to establish H2Bub1 and subsequent H3K4me and H3K79me in the gene body (Fig. 1; Xiao et al. 2005).

Although initially identified in yeast, the association of Rad6 with PAF and the elongating Pol II complex was found to be a highly conserved. Specifically, it was shown that the human PAF complex (hPAF) interacts

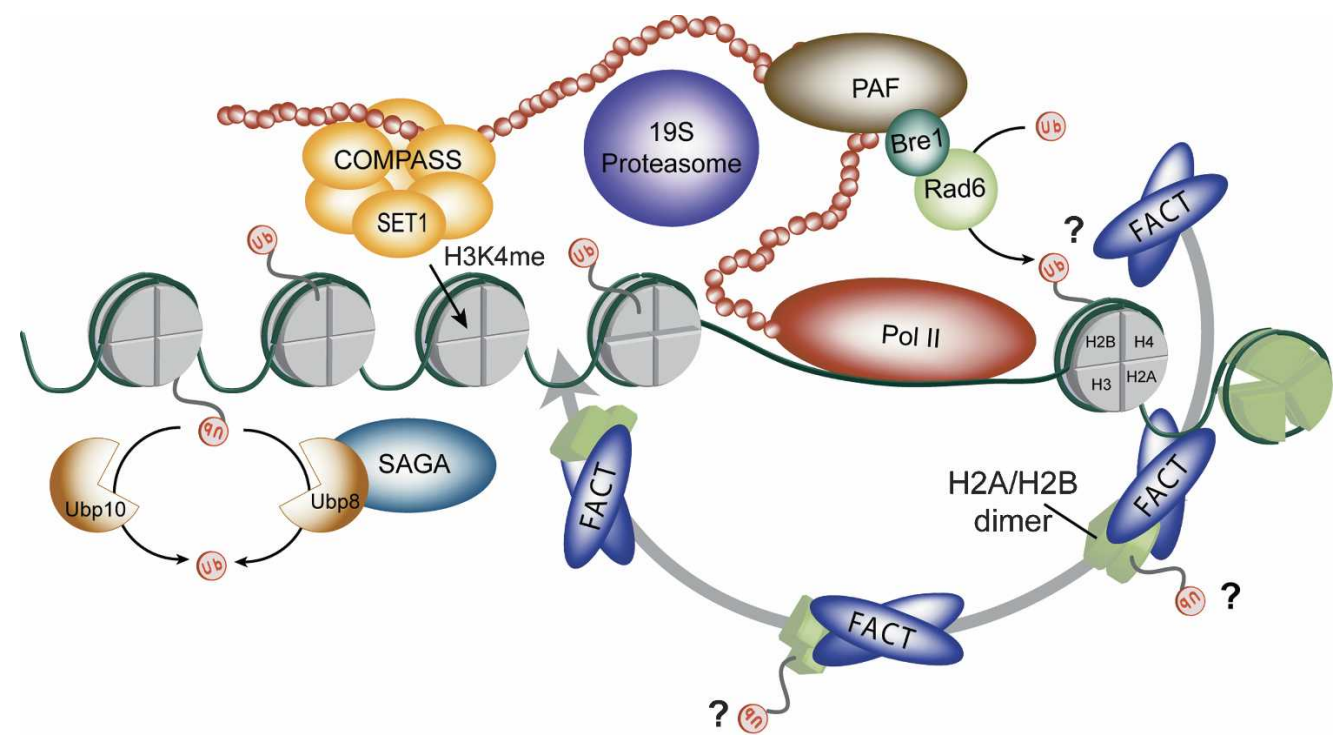

Figure 1. Role of Н2B ubiquitylation in transcript elongation. Ubiquitin is transferred to histone H2B by Rad6/Bre1, which is recruited to Pol II via the PAF transcription elongation complex that also stimulates Rad6's activity. The presence of H2Bub1 then recruits other activities (i.e., 19S proteasome) to transcribed genes and facilitates the function of other complexes on chromatin (i.e., COMPASS). During transcription elongation, H2Bub1 stimulates H2A/H2B dimer removal by FACT and facilitates efficient Pol II elongation through chromatin. While important for Pol II elongation, H2Bub1 is highly dynamic and must be removed through the activity of either SAGA-associated Ubp8 or by Ubp10 (independent of SAGA) to create correct histone methylation patterns within the gene body (not shown). The question marks in the figure represent the possibility that FACT selectively associates with ubiquitylated H2B-H2A dimers during the nucleosome disruption/reassembly process. Such disruption and coordination with the proteasome (see text) may promote the establishment of histone methylation "marks." 
both with Pol II and with the E2 enzyme UbcH6 and the E3 ligases RNF20/40 to establish H2Bub1 and regulate gene expression, most notably HOX genes (Zhu et al. 2005). Together with the fact that the BUR transcription elongation complex, the CTD of Pol II, and Kin28 (the kinase in TFIIH that regulates the initiation events of elongation) regulate $\mathrm{H} 2 \mathrm{Bub1}$, the data in yeast and human cells illustrate that H2Bub1 is a histone "mark" intimately linked to Pol II transcription (Laribee et al. 2005; Wood et al. 2005; Xiao et al. 2005).

The identification of H2Bub1 as an elongation-linked histone modification suggested that a good proportion of the total pool of H2B should carry this modification, especially in yeast, where the majority of the genome is transcriptionally active. How then does the cell maintain such low levels of H2Bub1? A solution for this problem presented itself in the form of an ubiquitin protease (Ubp8) that was identified as a functional subunit of the SAGA histone acetyltransferase complex (Henry et al. 2003; Daniel et al. 2004). This enzyme was found to catalyze H2B deubiquitylation on SAGA-dependent genes and to be important for establishing correct histone methylation patterns for H3K4me and H3 Lys 36 methylation (H3K36me) (Henry et al. 2003; Daniel et al. 2004). Deletion of Ubp8 resulted in transcription defects on SAGA-regulated genes, suggesting that $\mathrm{H} 2 \mathrm{~B}$ is normally ubiquitylated and then deubiquitylated to promote gene transcription. A second ubiquitin protease, Ubp10, also was found to deubiquitylate H2B. This enzyme acts primarily, although not exclusively, at sites of silent chromatin to maintain low levels of H2Bub1 and $\mathrm{H} 3 \mathrm{~K} 4 \mathrm{me} / \mathrm{H} 3 \mathrm{~K} 79 \mathrm{me}$, thereby promoting the maintenance of transcriptionally repressive chromatin states (Emre et al. 2005; Gardner et al. 2005). Deubiquitylation by Ubp 8 and Ubp10 has been proposed to account for all of the active removal of H2Bub1 (Gardner et al. 2005). Collectively, these studies reveal that $\mathrm{H} 2 \mathrm{~B}$ ubiquitylation and deubiquitylation is important for transcriptional activity and for establishing correct histone methylation patterns within the gene body (Fig. 1).

\section{FACT or fiction-a direct role for H2B monoubiquitylation in regulating transcription elongation?}

While numerous studies have identified how H2Bub1 becomes established on chromatin, significantly less progress has been made in understanding the actual role of H2Bub1 in the transcription process. The identification of the "trans-tail" pathway described above suggested that at least one role for H2Bub1 would be to establish downstream chromatin modifications that promote transcription. Recent studies into the function of H3K4me3 would support this idea. This modification has been linked to the direct recruitment of multiple different types of chromatin-modifying activities, including ATP-dependent chromatin remodeling factors (i.e., CHD1, NURF), histone acetylases/deacetylases (i.e., ING proteins), and even a histone demethylase (i.e., JMJD2A) (Ruthenburg et al. 2007). All of these activities could significantly impact transcription elongation by modulating a gene's chromatin conformation, which would either facilitate or repress Pol II's capacity to transcribe the chromatin template.

However, a recent report by Pavri et al. (2006) in Cell suggests that, while $\mathrm{H} 2 \mathrm{Bub} 1$ has a significant regulatory role in transcription elongation, this elongation function is not mediated by H3K4me. In vitro transcription elongation assays were used to establish that the dimeric FACT (facilitates chromatin transcription) complex could stimulate low levels of activator-dependent transcription through a chromatinized template; however, robust transcription elongation required the presence of PAF, Ubc6H, RNF20/40, and ubiquitin. Furthermore, it was found that FACT activity promoted $\mathrm{H} 2 \mathrm{~B}$ ubiquitylation and that FACT itself was stimulated by H2Bub1. Studies in budding yeast confirm both a genetic and functional interaction between FACT and H2Bub1 in vivo, lending support to the idea that FACT and $\mathrm{H} 2 \mathrm{Bub} 1$ collaborate in transcriptional regulation (M.A. Osley, pers. comm.). FACT is proposed to act during transcription elongation to remove a single $\mathrm{H} 2 \mathrm{~A} / \mathrm{H} 2 \mathrm{~B}$ dimer from nucleosomes to form hexasomes, which decreases the nucleosomal barrier to the elongating polymerase (Fig. 1; Reinberg and Sims 2006). In addition, FACT is also involved in nucleosome reassembly during the elongation process (Kaplan et al. 2003; Mason and Struhl 2003). Interestingly, time-course experiments done in vitro using this defined elongation assay and in vivo, using RNA interference (RNAi) in mammalian cells to knock down expression of factors involved in H2Bub1, show that loss of H2Bub1 has a severe defect in the kinetics of transcription but, over time, transcription occurs even in the absence of H2Bub1 (Pavri et al. 2006). These results are consistent with data in budding yeast, showing that deletion of factors that establish H2Bub1 is tolerated but leads to significant reductions in transcription kinetics (Xiao et al. 2005).

Contrary to the positive effect that H2Bub1 had on elongation, H3K4me had no detectable effect in this same study using the in vitro chromatinized transcription assay (Pavri et al. 2006). These results were interpreted to mean that, while $\mathrm{H} 2 \mathrm{Bub} 1$ promotes the removal of a H2A/H2B dimer by FACT to facilitate Pol II elongation, H3K4me is not involved in this process. Because of the prerequisite for H2Bub1 in the establishment of H3K4me and the tight correlation between the occurrence of H3K4me3 and transcriptionally active genes, an obvious question to ask is: What is the function of H3K4me3 in transcription? Because this study used an in vitro reconstituted chromatinized template, Pavri et al. (2006) were able to identify the minimal set of factors needed to transcribe this template. How reflective this in vitro system is of transcription occurring in vivo is not known, and thus one possibility could be that H3K4me3 regulates an aspect of transcription that was not tested in this assay. For example, in vivo chromatin forms higher-order, compacted structures that inherently represses transcription. This repressive chromatin structure must be contended with in order to transcribe 
genes. H3K4me3 may alleviate this repressive state by recruiting factors that act to open up compacted chromatin, such as ATP-dependent chromatin remodeling factors and histone acetyltransferases (see above), thus promoting decompaction of the chromatin fiber beyond what would occur solely through the activities of FACT (Ruthenburg et al. 2007). Another possibility suggested in this study is that $\mathrm{H} 3 \mathrm{~K} 4 \mathrm{me} 3$ may coordinate mRNA processing functions necessary for the generation of correct mRNAs (Pavri et al. 2006). Given the restriction of H3K4me3 to the 5' ends of actively transcribed genes, it would not be surprising to find that mRNA processing factors that associate with transcriptionally active genes may have subunits that interact with modified histones, in particular $\mathrm{H} 3 \mathrm{~K} 4 \mathrm{me} 3$. Tethering these factors to the $5^{\prime}$ ends of genes via $\mathrm{H} 3 \mathrm{~K} 4 \mathrm{me} 3$ may put them in an ideal location to interact with the nascent transcript or to be physically loaded onto the elongating Pol II complex. Whatever the role of $\mathrm{H} 3 \mathrm{~K} 4 \mathrm{me}$ in the transcription process, it does not appear to impact significantly on Pol II's ability to elongate through chromatin.

In this issue of Genes \& Development, Tanny et al. (2007) present in vivo data from Schizosacchromyces pombe (fission yeast) that also links H2Bub1 to an active role in transcription elongation independent of $\mathrm{H} 3 \mathrm{~K} 4 \mathrm{me}$. In this study, Tanny et al. (2007) find that loss of $\mathrm{H} 2 \mathrm{Bub1}$, either through mutation of the site of monoubiquitylation on $\mathrm{H} 2 \mathrm{~B}(K 119 R)$ or through deleting $S$. pombe homologs of the $\mathrm{H} 2 \mathrm{~B}$ monoubiquitylation factors, results in loss of $\mathrm{H} 3 \mathrm{~K} 4 \mathrm{me}$ (consistent with "transtail" pathway established in budding yeast) and a slow growth phenotype that involves defects in cell septation. By deleting the enzyme that methylates H3K4 (Set1), Tanny et al. (2007) show that the growth defects associated with the $K 119 R$ mutation come not from the loss of $\mathrm{H} 3 \mathrm{~K} 4 \mathrm{me}$ (since set1 $\Delta$ has no effect) but instead are due to other consequences of H2Bub1. To understand the contribution that $\mathrm{H} 2 \mathrm{Bub} 1$ makes in transcriptional regulation versus that made by $\mathrm{H} 3 \mathrm{~K} 4 \mathrm{me}$, gene expression array experiments were performed in mutants lacking H2Bub1 (htb1-K119R) or mutants lacking H3K4me (set1s). A much higher number of genes were shown to reproducibly alter their transcriptional profile (genes that either increase or decrease in expression) in $h t b 1-K 119 R$ relative to the set $1 \Delta$ strain $(84$ genes in the H2Bub1 mutants compared with 12 genes in set1 $1 \Delta)$. Of the genes in the set $1 \Delta$ strain that consistently altered expression, 50\% were shared with the $h t b 1-K 119 R$ mutant, consistent with H2Bub1's role in establishing H3K4me. These results argue that $\mathrm{H} 2 \mathrm{Bubl}$ has a much greater effect on transcription in vivo than does $\mathrm{H} 3 \mathrm{~K} 4 \mathrm{me}$.

To address this issue more directly, Tanny et al. (2007) compared the distribution of Pol II on two genes (hem2 $2^{+}$ and $\left.\operatorname{sod} 2^{+}\right)$that are repressed in both the $h t b 1-K 119 R$ and set $1 \Delta$ mutants. The aro $1^{+}$gene also was examined as a control since expression of this gene remained unchanged in the microarray experiments performed. They found that in the $h t b 1-K 119 R$ mutant, an overall decrease in the level of Pol II at the $3^{\prime}$ end relative to the $5^{\prime}$ end occurred compared with wild type on both hem $2^{+}$ and $\operatorname{sod} 2^{+}$, but not aro $^{+}$, and this change in Pol II density correlated with the decrease in transcription of these genes. The change in Pol II density was associated with alterations in the chromatin configuration of $\operatorname{sod} 2^{+}$, but not hem2 $2^{+}$or aro $^{+}$, since the density of histone H3 increased near the $5^{\prime}$ end of the gene relative to that found at the $3^{\prime}$ end in the $h t b 1-K 119 R$ mutant. This effect also was seen in the $\operatorname{set} 1 \Delta$ strain but to a significantly lesser extent. These results suggested that H2Bub1 might be involved in a chromatin assembly pathway that is coupled to transcription. To test this genetically, the $h t b 1-K 119 R$ mutant was crossed to deletion mutants of the H2A.Z histone variant (pht1s) or to a deletion mutant of the histone chaperone HIRA (hip1s). Tetrad analysis of the crosses found no double mutants whereas a set $1 \Delta$ pht $1 \Delta$ or a set $1 \Delta$ hip $1 \Delta$ double mutant could readily be generated. These results indicate that the combination of the $h t b 1-K 119 R$ mutant with either loss of the H2A.Z variant or with HIRA is synthetically lethal. Interestingly, in budding yeast, an $h t z 1 \Delta$ (the H2A.Z variant) combined with mutants in either COMPASS or Rad6 is synthetically lethal (Krogan et al. 2003). Nevertheless, the genetic data in $S$. pombe are consistent with the hypothesis that H2Bub1 is linked directly to a pathway that mediates chromatin assembly/disassembly. Whether this pathway is FACT mediated or involves other histone chaperones remains to be determined.

\section{The proteasome and $\mathrm{H} 2 \mathrm{Bub1}$-tweaking elongation?}

Monoubiquitylation of $\mathrm{H} 2 \mathrm{~B}$ has a relatively unique role in chromatin biology given its functions in controlling downstream histone modifications and stimulating transcription-dependent chromatin assembly/disassembly mediated by FACT. What else does this multitalented, transient histone modification do? One exciting consequence of H2Bub1 involves recruitment of the 19S proteasome to genes. The $19 \mathrm{~S}$ proteasome is a known transcriptional regulator that associates with the promoter, coding, and 3' end of genes (Collins and Tansey 2006). While it participates in transcription initiation, elongation, and transcript termination, how it regulates these processes remains poorly understood. New insight into 19S function reveals that the chaperone activity of this complex is required for the establishment of H3K4me and H3K79me and that the recruitment of the 19S to genes depends on H2Bub1 (Ezhkova and Tansey 2004). Thus, the 19S is the first complex identified that can uncouple $\mathrm{H} 2 \mathrm{Bub} 1$ regulation from $\mathrm{H} 3 \mathrm{~K} 4 \mathrm{me}$ and $\mathrm{H} 3 \mathrm{~K} 79$ me regulation (thereby providing an important downstream effector in the "trans-tail" pathway). How the $19 \mathrm{~S}$ regulates histone methylation is unknown, but a recent study showing that the ATPase activity of the 19S can alter the SAGA complex to enhance its interactions with transcriptional activators and regulate histone $\mathrm{H} 3$ acetylation suggests that the $19 \mathrm{~S}$ particle might act directly on histone methyltransferases and their associated factors to promote methylation (Lee et al. 2005). The identification of the $19 \mathrm{~S}$ proteasome as downstream of H2Bub1 significantly widens the sphere of influence that 
this histone modification has on the transcription process and leads to the possibility that it may have an unrealized role in other aspects of transcriptional regulation (see below).

\section{Future questions}

The connections made between H2Bub1 and FACT, PAF, SAGA, and the 19S complex demonstrate the importance of this modification in the transcription elongation process. Although considerable progress in understanding the regulation of this histone mark and significant inroads into its effector functions have been made, we would argue that complete understanding of H2Bub1's role in transcription lies ahead. For the purpose of stimulating future research into H2Bub1's functions, we propose a series of questions we believe are some of the pressing issues remaining to be resolved.

(1) What is the role of H2Bub1 in the promoter regions of genes? H2Bub1 can be readily detected within the promoter regions of transcriptionally active genes yet promoter-specific functions have not been ascribed to it (Henry et al. 2003; Kao et al. 2004; Xiao et al. 2005). Does it act at the promoter solely to establish H3K4me3, or does it also have other functions such as in preinitiation complex assembly and/or coactivator recruitment? Notably, FACT actively promotes formation of transcription initiation complexes by promoting binding of TBP to TATA sequences in vitro and in vivo (Biswas et al. 2005). Given the linkage between H2Bubl and FACT function discussed above, an intriguing hypothesis for H2Bub1 could be that it stimulates FACT recruitment to promoter regions to promote TBP binding and formation of initiation complexes.

(2) Does H2Bub1 selectively mark H2A/H2B dimers for removal by FACT? While FACT actively removes a $\mathrm{H} 2 \mathrm{~A} / \mathrm{H} 2 \mathrm{~B}$ dimer to promote transcription elongation, it is unclear whether the dimer removed is physically "marked" by H2Bub1. One possibility may be that the stimulation of FACT activity by H2Bub1 is because FACT preferentially associates with ubiquitylated H2A/ H2B dimers (see Fig. 1). In this scenario, H2Bub1 functions to enhance association of FACT on genes, which would further stimulate FACT's activity in removing these same dimers (or neighboring ones) during Pol II elongation. Another possibility might be that FACT preferentially interacts with nucleosomes containing H2Bub1, but the dimer removal activity of FACT is not dependent on the H2B modification. Either case could be possible, but only future biochemical studies will dissect the precise link between FACT and H2Bub1.

(3) How does H2Bub1 recruit the 19S proteasome and does it function in conjunction with FACT? While 19S recruitment to genes depends on the presence of H2Bub1, whether components of the $19 \mathrm{~S}$ bind directly to the modification or are recruited via another factor that is dependent on H2Bub1 remains uncertain. Subunits within the 19S proteasome may have the potential to directly recognize the ubiquitin mark (Pickart 2001). Another possibility could be that elongation factors that require $\mathrm{H} 2 \mathrm{Bub} 1$ for their recruitment or function, especially FACT, recruit the 19S. Previous studies have shown an association between FACT and the 19S; however, the functional significance of this interaction remains unclear (Ferdous et al. 2001). Identifying how H2Bub1 recruits the 19S proteasome to genes and then further pursuing how the 19S functions in the transcription process will ultimately elucidate the central role of H2Bub1 in 19S and FACT activity on genes.

(4) Is H2Bub1 a transient mark that occurs chiefly in the initial rounds of gene transcription? Mounting evidence has established a strong link between FACT and H2Bub1 in regulating proper Pol II elongation through chromatin. However, it is unclear why Rad6 and H2Bub1 are only present during the early time periods of gene activation but not detectable during sustained gene transcription (Kao et al. 2004; Xiao et al. 2005). One idea to explain this quandary may be that newly transcribed chromatin is highly compact and may need additional mechanisms to disrupt chromatin during the first round(s) of transcription. In this case, H2Bub1 may act as both a "wedge" and a "bridge" by helping to facilitate the unfolding of the compact chromatin fiber that surrounds an untranscribed gene while at the same time stabilizing FACT binding to facilitate $\mathrm{H} 2 \mathrm{~A} / \mathrm{H} 2 \mathrm{~B}$ dimer removal (Henry and Berger 2002). After the initial passage of Pol II facilitated by H2Bub1, the disrupted chromatin state may be maintained by subsequent passages of the Pol II complex in a type of "self-perpetuating" mechanism. Thus, maintenance of this disrupted state may no longer require H2Bub1 even though FACT would be required during each round of transcription since a constant level of nucleosome disruption/reassembly is required for Pol II elongation in chromatin. Future in vivo studies aimed at examining the precise organization of chromatin surrounding genes during the elongation process will likely determine if H2Bub1 contributes to "unraveling" higher-order chromatin structure during the initial stages of gene activation.

(5) Does H2Bub1 regulate mRNA processing? Because the $19 \mathrm{~S}$ proteasome has roles in transcription termination, decreased recruitment of this complex due to loss of H2Bub1 might affect the generation of mature mRNAs (Gillette et al. 2004). Furthermore, defects in the PAF complex, a critical regulator of H2Bub1, result in the production of mRNAs with shortened poly(A) tails (Mueller et al. 2004). Are any of these defects, either in termination or mRNA poly(A) tail length, due to the loss of H2Bub1? Only by testing for altered mRNAs in mutants defective for H2Bub1 will we be able to unequivocally identify a role, if any, for this modification in mRNA processing.

These questions by no means reflect the only important issues remaining to be addressed for understanding the role of H2Bub1 in chromatin biology. However, we believe that they will focus attention on key aspects of H2B ubiquitylation's function in transcription for future exploration. Most of the characterization of H2Bub1 function has occurred in the past 7 years. We look forward to seeing what the next 7 years have in store. 


\section{Acknowledgments}

We thank Mary Ann Osley and Zu-Wen Sun for communicating unpublished results and for critically evaluating this paper. B.D.S and R.N.L. are supported by grants from the National Institutes of Health. S.M.F. is supported by a UNC Lineberger Comprehensive Cancer Center training grant. B.D.S. is a Pew Scholar in the Biomedical Sciences.

\section{References}

Bernstein, B.E., Humphrey, E.L., Erlich, R.L., Schneider, R., Bouman, P., Liu, J.S., Kouzarides, T., and Schreiber, S.L. 2002. Methylation of histone H3 Lys 4 in coding regions of active genes. Proc. Natl. Acad. Sci. 99: 8695-8700.

Biswas, D., Yu, Y., Prall, M., Formosa, T., and Stillman, D.J. 2005. The yeast FACT complex has a role in transcriptional initiation. Mol. Cell. Biol. 25: 5812-5822.

Briggs, S.D., Bryk, M., Strahl, B.D., Cheung, W.L., Davie, J.K., Dent, S.Y., Winston, F., and Allis, C.D. 2001. Histone H3 lysine 4 methylation is mediated by Set1 and required for cell growth and rDNA silencing in Saccharomyces cerevisiae. Genes \& Dev. 15: 3286-3295.

Briggs, S.D., Xiao, T., Sun, Z.W., Caldwell, J.A., Shabanowitz, J., Hunt, D.F., Allis, C.D., and Strahl, B.D. 2002. Gene silencing: Trans-histone regulatory pathway in chromatin. Nature 418: 498.

Brownell, J.E., Zhou, J., Ranalli, T., Kobayashi, R., Edmondson, D.G., Roth, S.Y., and Allis, C.D. 1996. Tetrahymena histone acetyltransferase A: A homolog to yeast Gen5p linking histone acetylation to gene activation. Cell 84: 843-851.

Bryk, M., Briggs, S.D., Strahl, B.D., Curcio, M.J., Allis, C.D., and Winston, F. 2002. Evidence that Set1, a factor required for methylation of histone $\mathrm{H} 3$, regulates rDNA silencing in $S$. cerevisiae by a Sir2-independent mechanism. Curr. Biol. 12: 165-170.

Collins, G.A. and Tansey, W.P. 2006. The proteasome: A utility tool for transcription? Curr. Opin. Genet. Dev. 16: 197-202.

Daniel, J.A., Torok, M.S., Sun, Z.W., Schieltz, D., Allis, C.D., Yates III, J.R., and Grant, P.A. 2004. Deubiquitination of histone H2B by a yeast acetyltransferase complex regulates transcription. J. Biol. Chem. 279: 1867-1871.

Dover, J., Schneider, J., Tawiah-Boateng, M.A., Wood, A., Dean, K., Johnston, M., and Shilatifard, A. 2002. Methylation of histone $\mathrm{H} 3$ by COMPASS requires ubiquitination of histone H2B by Rad6. J. Biol. Chem. 277: 28368-28371.

Emre, N.C., Ingvarsdottir, K., Wyce, A., Wood, A., Krogan, N.J., Henry, K.W., Li, K., Marmorstein, R., Greenblatt, J.F., Shilatifard, A., et al. 2005. Maintenance of low histone ubiquitylation by Ubp10 correlates with telomere-proximal Sir2 association and gene silencing. Mol. Cell 17: 585-594.

Ezhkova, E. and Tansey, W.P. 2004. Proteasomal ATPases link ubiquitylation of histone $\mathrm{H} 2 \mathrm{~B}$ to methylation of histone $\mathrm{H} 3$. Mol. Cell 13: 435-442.

Ferdous, A., Gonzalez, F., Sun, L., Kodadek, T., and Johnston, S.A. 2001. The $19 \mathrm{~S}$ regulatory particle of the proteasome is required for efficient transcription elongation by RNA polymerase II. Mol. Cell 7: 981-991.

Gardner, R.G., Nelson, Z.W., and Gottschling, D.E. 2005. Ubp10/Dot4p regulates the persistence of ubiquitinated histone H2B: Distinct roles in telomeric silencing and general chromatin. Mol. Cell. Biol. 25: 6123-6139.

Gillette, T.G., Gonzalez, F., Delahodde, A., Johnston, S.A., and Kodadek, T. 2004. Physical and functional association of RNA polymerase II and the proteasome. Proc. Natl. Acad. Sci. 101: 5904-5909.
Henry, K.W. and Berger, S.L. 2002. Trans-tail histone modifications: Wedge or bridge? Nat. Struct. Biol. 9: 565-566.

Henry, K.W., Wyce, A., Lo, W.S., Duggan, L.J., Emre, N.C., Kao, C.F., Pillus, L., Shilatifard, A., Osley, M.A., and Berger, S.L. 2003. Transcriptional activation via sequential histone H2B ubiquitylation and deubiquitylation, mediated by SAGA-associated Ubp8. Genes \& Dev. 17: 2648-2663.

Hwang, W.W., Venkatasubrahmanyam, S., Ianculescu, A.G., Tong, A., Boone, C., and Madhani, H.D. 2003. A conserved RING finger protein required for histone $\mathrm{H} 2 \mathrm{~B}$ monoubiquitination and cell size control. Mol. Cell 11: 261-266.

Kao, C.F., Hillyer, C., Tsukuda, T., Henry, K., Berger, S., and Osley, M.A. 2004. Rad6 plays a role in transcriptional activation through ubiquitylation of histone H2B. Genes \& Dev. 18: 184-195.

Kaplan, C.D., Laprade, L., and Winston, F. 2003. Transcription elongation factors repress transcription initiation from cryptic sites. Science 301: 1096-1099.

Kim, M., Ahn, S.H., Krogan, N.J., Greenblatt, J.F., and Buratowski, S. 2004. Transitions in RNA polymerase II elongation complexes at the $3^{\prime}$ ends of genes. EMBO J. 23: 354-364.

Krogan, N.J., Kim, M., Ahn, S.H., Zhong, G., Kobor, M.S., Cagney, G., Emili, A., Shilatifard, A., Buratowski, S., and Greenblatt, J.F. 2002. RNA polymerase II elongation factors of Saccharomyces cerevisiae: A targeted proteomics approach. Mol. Cell. Biol. 22: 6979-6992.

Krogan, N.J., Keogh, M.C., Datta, N., Sawa, C., Ryan, O.W., Ding, H., Haw, R.A., Pootoolal, J., Tong, A., Canadien, V., et al. 2003. A Snf2 family ATPase complex required for recruitment of the histone H2A variant Htz1. Mol. Cell 12: 15651576.

Laribee, R.N., Krogan, N.J., Xiao, T., Shibata, Y., Hughes, T.R., Greenblatt, J.F., and Strahl, B.D. 2005. BUR kinase selectively regulates $\mathrm{H} 3 \mathrm{~K} 4$ trimethylation and $\mathrm{H} 2 \mathrm{~B}$ ubiquitylation through recruitment of the PAF elongation complex. Curr. Biol. 15: 1487-1493.

Lee, D., Ezhkova, E., Li, B., Pattenden, S.G., Tansey, W.P., and Workman, J.L. 2005. The proteasome regulatory particle alters the SAGA coactivator to enhance its interactions with transcriptional activators. Cell 123: 423-436.

Mason, P.B. and Struhl, K. 2003. The FACT complex travels with elongating RNA polymerase II and is important for the fidelity of transcriptional initiation in vivo. Mol. Cell. Biol. 23: 8323-8333.

Mizzen, C.A., Yang, X.J., Kokubo, T., Brownell, J.E., Bannister, A.J., Owen-Hughes, T., Workman, J., Wang, L., Berger, S.L., Kouzarides, T., et al. 1996. The TAF 250 subunit of TFIID has histone acetyltransferase activity. Cell 87: 1261-1270.

Mueller, C.L. and Jaehning, J.A. 2002. Ctr9, Rtf1, and Leo1 are components of the Pafl/RNA polymerase II complex. Mol. Cell. Biol. 22: 1971-1980.

Mueller, C.L., Porter, S.E., Hoffman, M.G., and Jaehning, J.A 2004. The Paf1 complex has functions independent of actively transcribing RNA polymerase II. Mol. Cell 14: 447456.

Ng, H.H., Xu, R.M., Zhang, Y., and Struhl, K. 2002. Ubiquitination of histone H2B by Rad6 is required for efficient Dot1mediated methylation of histone $\mathrm{H} 3$ lysine 79. J. Biol. Chem. 277: 34655-34657.

Ng, H.H., Dole, S., and Struhl, K. 2003. The Rtf1 component of the Paf1 transcriptional elongation complex is required for ubiquitination of histone H2B. J. Biol. Chem. 278: 3362533628.

Osley, M.A. 2004. H2B ubiquitylation: The end is in sight. Biochim. Biophys. Acta 1677: 74-78.

Pavri, R., Zhu, B., Li, G., Trojer, P., Mandal, S., Shilatifard, A., 
and Reinberg, D. 2006. Histone H2B monoubiquitination functions cooperatively with FACT to regulate elongation by RNA polymerase II. Cell 125: 703-717.

Pickart, C.M. 2001. Mechanisms underlying ubiquitination. Annu. Rev. Biochem. 70: 503-533.

Reinberg, D. and Sims III, R.J. 2006. de FACTo nucleosome dynamics. J. Biol. Chem. 281: 23297-23301.

Robzyk, K., Recht, J., and Osley, M.A. 2000. Rad6-dependent ubiquitination of histone H2B in yeast. Science 287: 501504.

Ruthenburg, A.J., Allis, C.D., and Wysocka, J. 2007. Methylation of lysine 4 on histone H3: Intricacy of writing and reading a single epigenetic mark. Mol. Cell 25: 15-30.

Santos-Rosa, H., Schneider, R., Bannister, A.J., Sherriff, J., Bernstein, B.E., Emre, N.C., Schreiber, S.L., Mellor, J., and Kouzarides, T. 2002. Active genes are tri-methylated at K4 of histone H3. Nature 419: 407-411.

Sun, Z.W. and Allis, C.D. 2002. Ubiquitination of histone H2B regulates $\mathrm{H} 3$ methylation and gene silencing in yeast. $\mathrm{Na}$ ture 418: 104-108.

Tanny, J.C., Erdjument-Bromage, H., Tempst, P., and Allis, C.D. 2007. Ubiquitylation of histone H2B controls RNA polymerase II transcription elongation independently of histone $\mathrm{H} 3$ methylation. Genes \& Dev. (this issue).

van Leeuwen, F., Gafken, P.R., and Gottschling, D.E. 2002. Dotlp modulates silencing in yeast by methylation of the nucleosome core. Cell 109: 745-756.

Wood, A., Krogan, N.J., Dover, J., Schneider, J., Heidt, J., Boateng, M.A., Dean, K., Golshani, A., Zhang, Y., Greenblatt, J.F., et al. 2003a. Bre1, an E3 ubiquitin ligase required for recruitment and substrate selection of Rad6 at a promoter. Mol. Cell 11: 267-274.

Wood, A., Schneider, J., Dover, J., Johnston, M., and Shilatifard, A. 2003b. The Paf1 complex is essential for histone monoubiquitination by the Rad6-Brel complex, which signals for histone methylation by COMPASS and Dotlp. I. Biol. Chem. 278: 34739-34742.

Wood, A., Schneider, J., Dover, J., Johnston, M., and Shilatifard, A. 2005. The Bur1/Bur2 complex is required for histone H2B monoubiquitination by Rad6/Bre1 and histone methylation by COMPASS. Mol. Cell 20: 589-599.

Xiao, T., Kao, C.F., Krogan, N.J., Sun, Z.W., Greenblatt, J.F., Osley, M.A., and Strahl, B.D. 2005. Histone H2B ubiquitylation is associated with elongating RNA polymerase II. Mol. Cell. Biol. 25: 637-651.

Zhang, Y. 2003. Transcriptional regulation by histone ubiquitination and deubiquitination. Genes \& Dev. 17: 2733-2740.

Zhu, B., Zheng, Y., Pham, A.D., Mandal, S.S., Erdjument-Bromage, H., Tempst, P., and Reinberg, D. 2005. Monoubiquitination of human histone H2B: The factors involved and their roles in HOX gene regulation. Mol. Cell 20: 601-611. 


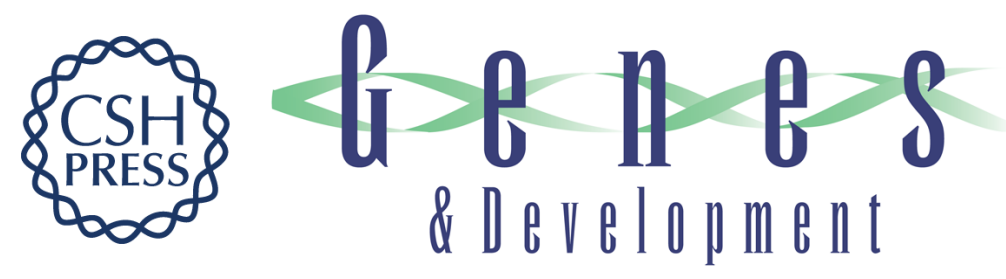

\section{H2B ubiquitylation in transcriptional control: a FACT-finding mission}

R. Nicholas Laribee, Stephen M. Fuchs and Brian D. Strahl

Genes Dev. 2007, 21:

Access the most recent version at doi:10.1101/gad.1541507

\section{Related Content Ubiquitylation of histone H2B controls RNA polymerase II transcription elongation independently of histone $\mathrm{H} 3$ methylation \\ Jason C. Tanny, Hediye Erdjument-Bromage, Paul Tempst, et al. \\ Genes Dev. April , 2007 21: 835-847}

References This article cites 45 articles, 21 of which can be accessed free at:

http://genesdev.cshlp.org/content/21/7/737.full.html\#ref-list-1

Articles cited in:

http://genesdev.cshlp.org/content/21/7/737.full.htmI\#related-urls

\section{License}

Email Alerting Receive free email alerts when new articles cite this article - sign up in the box at the top Service right corner of the article or click here.

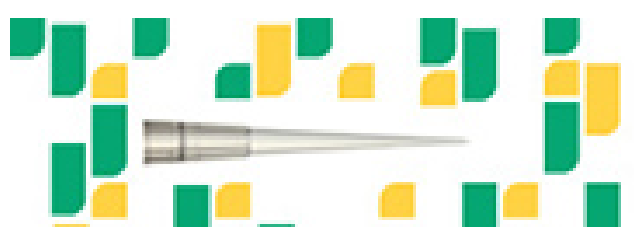

Focused on your science. 